\title{
Review \\ Bench-to-bedside review: Current evidence for extracorporeal albumin dialysis systems in liver failure \\ Constantine J Karvellas ${ }^{1,2}$, Noel Gibney ${ }^{3}$, Demetrios Kutsogiannis ${ }^{3}$, Julia Wendon ${ }^{2}$ \\ and Vincent G Bain ${ }^{1}$
}

\author{
1 University of Alberta Liver Unit, Zeidler-Ledcor Building, 130 University Campus, Edmonton, Alberta, T6G 2X8 Canada \\ 2Institute of Liver Studies, King's College Hospital, Denmark Hill, London, SE5 9RS, UK \\ ${ }^{3}$ Division of Critical Care Medicine, University of Alberta, 3C1 Walter C Mackenzie Health Sciences Centre, 8440-112 Street, Edmonton, Alberta \\ T6G 2B7, Canada
}

Corresponding author: Vincent G Bain, vince.bain@ualberta.ca

Published: 8 June 2007

Critical Care 2007, 11:215 (doi:10.1186/cc5922)

This article is online at http://ccforum.com/content/11/3/215

(c) 2007 BioMed Central Ltd

\begin{abstract}
Acute liver failure (ALF) and acute on chronic liver failure (AoCLF) carry a high mortality. The rationale for extracorporeal systems is to provide an environment facilitating recovery or a window of opportunity for liver transplantation. Recent technologies have used albumin as a scavenging molecule. Two different albumin dialysis systems have been developed using this principle: MARS (Molecular Adsorbent Recirculation System) and SPAD (SinglePass Albumin Dialysis). A third system, Prometheus (Fractionated Plasma Separation and Adsorption), differs from the others in that the patient's albumin is separated across a membrane and then is run over adsorptive columns. Although several trials have been published (particularly with MARS), currently there is a lack of controlled studies with homogenous patient populations. Many studies have combined patients with ALF and AoCLF. Others have included patients with different etiologies. Although MARS and Prometheus have shown biochemical improvements in AoCLF and ALF, additional studies are required to show conclusive benefit in short- and long-term survival. The appropriate comparator is standard medical therapy rather than head-to-head comparisons of different forms of albumin dialysis.
\end{abstract}

\section{Introduction}

Acute liver failure (ALF) and acute on chronic liver failure (AoCLF) have a high mortality. ALF is defined as development of severe acute liver injury with impaired hepatic synthetic function and encephalopathy in a patient without previous liver disease [1]. AoCLF is defined as acute deterioration in liver function over a 2- to 4-week period in a patient with preexisting chronic liver disease. In both conditions, the lack of the metabolic and regulatory function of the liver results in life-threatening complications that may include bleeding, renal failure, hepatic encephalopathy (HE) or cerebral edema, cardiovascular failure, and susceptibility to infections culminating in multi-organ failure [2].

Currently, the only effective therapy for patients with liver failure is liver transplantation. Many patients die, however, before a suitable graft is available, and for those who progress to multi-organ failure, transplantation is not an option. The liver often maintains some regenerative capacity, so the rationale for supportive therapy and extracorporeal systems is to provide an environment facilitating recovery to create or prolong a window of opportunity for liver transplantation or, in the best-case scenario, until native liver recovery occurs in ALF or a period of stability for those with AoCLF [3].

In liver failure, a variety of 'toxins' accumulate as a result of impaired hepatic function and clearance. Ammonia, inflammatory cytokines, aromatic amino acids, and endogenous benzodiazepines have been implicated in the development of $\mathrm{HE}$ and cerebral edema. Other systemic factors such as nitric oxide and cytokines have been linked with circulatory and renal dysfunction in liver failure. Pro-inflammatory cytokines and oxidative stress have broad effects ranging from increased capillary permeability to modulating cell-death and immune dysregulation.

Various forms of dialysis were used in the treatment of liver failure in the 1970 s but failed to show benefit, possibly because the majority of toxins in liver failure are waterinsoluble and albumin-bound and are poorly cleared by

$\mathrm{ALF}=$ acute liver failure; $\mathrm{AoCLF}=$ acute on chronic liver failure; $\mathrm{CBF}=$ cerebral blood flow; $\mathrm{CVVHD}=$ continuous venovenous hemodialysis; $\mathrm{FPSA}=$ fractionated plasma separation and adsorption; HBV = hepatitis B virus; $\mathrm{HE}=$ hepatic encephalopathy; HRS = hepatorenal syndrome; IL = interleukin; MAP = mean arterial pressure; MARS = Molecular Adsorbent Recirculation System; SMT = standard medical therapy; SOFA = Sequential Organ Failure Assessment; SPAD = Single-Pass Albumin Dialysis; SVRI = systemic vascular resistance index; TNF- $\alpha=$ tumor necrosis factoralpha. 
Table 1

\begin{tabular}{|c|c|c|c|c|c|c|}
\hline \multirow[b]{2}{*}{ Study } & \multirow[b]{2}{*}{ Number } & \multirow[b]{2}{*}{ Controlled } & \multicolumn{3}{|c|}{ Improvements } & \multirow[b]{2}{*}{ Survival (30 days)a } \\
\hline & & & Biochemical & CVS & CNS & \\
\hline Stange et al. [7] & 13 & No & Yes & $\mathrm{N} / \mathrm{A}$ & Yes & $69 \%$ \\
\hline Schmidt et al. [10] & 8 & No & Yes & Yes & No & $50 \%$ \\
\hline Jalan et al. [37] & 8 & No & Yes & Yes & Yes & $50 \%$ \\
\hline Di Campli et al. [38] & 13 & No & Yes & N/A & Yes & $38 \%$ \\
\hline Mitzner et al. [8] & 13 & Yes & Yes & Yes & No & Yes (37.5\% versus $0 \%$ at 7 days) \\
\hline Heemann et al. [9] & 23 & Yes & Yes & Yes & Yes & Yes $(90 \%$ versus $55 \%)$ \\
\hline Sen et al. [11] & 18 & Yes & $Y_{e s}^{b}$ & No & Yes & No $(45 \%$ in both) \\
\hline Blei [16] & 70 & Yes & $\mathrm{N} / \mathrm{A}$ & $\mathrm{N} / \mathrm{A}$ & Yes & $\mathrm{N} / \mathrm{A}$ \\
\hline Laleman et al. [12] & 18 & Yes & $Y_{e s}{ }^{c}$ & Yes & $\mathrm{N} / \mathrm{A}$ & N/A \\
\hline
\end{tabular}

Biochemical improvements: statistically significant reduction in bilirubin, bile acids, creatinine, and ammonia. aPercentages indicate uncontrolled survival data; btrend did not reach statistical significance; cbilirubin and bile acids only. CNS, improvement in hemodynamic parameters (mean arterial pressure, heart rate, vasopressor requirements); CNS, decrease in hepatic encephalopathy grade (neurological improvement); N/A, not assessed.

conventional hemodialysis or hemofiltration systems [4]. Charcoal hemoperfusion was similarly ineffective in a controlled study, possibly in part due to biocompatibility issues [5].

Albumin administration has been shown to be beneficial in spontaneous bacterial peritonitis and hepatorenal syndrome (HRS) partly due to its ability to bind toxins [4]. Recent artificial liver support technologies have used albumin as a binding and scavenging molecule. Albumin dialysis involves dialyzing blood against an albumin-containing solution across a highly permeable high-flux membrane. The blood-bound toxins are cleared by diffusion and taken up by the binding sites of the albumin dialysate. Two different albumin dialysis systems have been developed using this principle: MARS (Molecular Adsorbent Recirculation System) and SPAD (Single-Pass Albumin Dialysis). Prometheus (Fractionated Plasma Separation and Adsorption, or FPSA) differs from the others in that the patient's plasma is separated across a membrane and then is run over adsorptive columns. In this review, we will examine the current evidence for the use of these three technologies in patients with liver failure and its complications.

\section{Materials and methods}

Relevant articles were found by electronic literature search from the National Institutes of Health (PubMed) database as well as bibliographies of recovered articles. We included fulllength articles written in English between 1999 and 2006. Articles were excluded if statistical comparisons ( $p$ values) were not provided versus baseline in uncontrolled studies or versus the control group in randomized studies. In the absence of randomized studies, our comments reflect a narrative review of the available literature.

\section{Results}

\section{Molecular Adsorbents Recirculation System}

MARS was originally developed by Stange and colleagues [6] (Teraklin AG, Rostock, Germany) in 1993. The system consists of a blood circuit, an albumin circuit, and a classic 'renal' circuit. Blood is dialysed across an albuminimpregnated high-flux dialysis membrane; $600 \mathrm{ml}$ of $20 \%$ human albumin in the albumin circuit acts as the dialysate. Albumin-bound toxins in blood are released to the membrane. These are subsequently picked up by albumin in the dialysate, which then undergoes hemodialysis/hemofiltration if required. The albumin dialysate is subsequently cleansed via passage across two sequential adsorbent columns containing activated charcoal and anion exchange resin. These columns remove most of the water-soluble and albumin-bound toxins. Because of the pore size of the membrane, substances with a molecular weight of more than $50 \mathrm{kDa}$, such as essential hormones and growth factors bound to albumin, are not removed [7].

Although there is a large amount of uncontrolled data about MARS use, only a few small controlled studies to date have examined the utility and safety of MARS in patients with complications of liver failure. Most have focused on improvements in biochemical profile, HE, and HRS. The benefit of MARS has been more clearly shown in patients with AoCLF (Table 1).

\section{Molecular Adsorbent Recirculation System and acute on chronic liver failure}

In 2000, Mitzner and colleagues [8] reported 13 patients with AoCLF and type 1 HRS treated with MARS. Patients received a mean of five treatments and did not receive vaso- 
pressors nor were any transplanted. The authors showed a $37.5 \%$ absolute survival benefit at day 7 versus $0 \%$ in controls. Significant decreases in creatinine and bilirubin were also noted in the MARS group.

Subsequently, Heemann and colleagues [9] randomly assigned 23 patients with AoCLF (19 were alcoholics) to MARS or standard medical therapy [SMT] (including dialysis if necessary). Inclusion criteria included bilirubin of greater than $340 \mu \mathrm{mol} / \mathrm{l}$, HE of greater than grade 2, and renal dysfunction. Patients in the MARS group received up to 10 treatments in the first 2 weeks. At day 30,11/12 patients in the MARS group were still alive, compared to only $6 / 11$ in the control group $(p<0.05)$. There were also statistically significant decreases in bilirubin (43\%) and bile acids (29\%) in the MARS group but not in the control group. A statistically significant increase in mean arterial pressure (MAP) $(p<0.05)$ as well as reductions in creatinine and HE grade $(p<0.06)$ were noted in the MARS group. Although improvements with MARS were significant compared to baseline, it is difficult to extrapolate these results beyond 30 days. This study was predominantly composed of patients with alcoholic cirrhosis with a superimposed acute injury related to either recent drinking or infection. Therefore, these results may not be applicable to other populations with different etiologies of cirrhosis.

\section{Molecular Adsorbent Recirculation System and}

hemodynamics in acute on chronic liver failure

Although early uncontrolled studies have shown improvement in MAP in patients with AoCLF on MARS, results have been mixed in larger trials. In 2001, Schmidt and colleagues [10] reported an uncontrolled study of eight patients with AoCLF who received a single 10-hour MARS treatment. Statistically significant increases over baseline in MAP and systemic vascular resistance index (SVRI) (both $p<0.05$ ) were noted. However, Sen and colleagues [11] were unable to reproduce these findings in their trial of 18 patients with alcohol-related AoCLF randomly assigned to receive MARS or SMT (including hemodialysis if indicated) over 7 days. This study also assessed the effect of MARS on cytokine profiles and nitric oxide, a potent vasodilator thought to have a significant effect on systemic vascular resistance in liver failure. Although HE improved significantly in the MARS group compared to control $(p<0.05)$, there were no statistically significant differences in cytokines, ammonia, malondialdehyde (marker of oxidative stress), or nitric oxide levels. There also was no statistically significant difference in MAP noted in either group from baseline nor mortality benefit at 30 days (4/9 alive in each group). The study of Schmidt and colleagues [10] assessed hemodynamics after one run on MARS, whereas the results of Sen and colleagues [11] were obtained after multiple MARS treatments.

Interestingly, a more recent study of 18 patients with AoCLF (alcoholic hepatitis) compared the hemodynamic effects of MARS versus Prometheus versus SMT (six patients in each arm; see next section) [12]. They showed statistically significant increases in MAP and SVRI and a decrease in endogenous vasoactive compounds with MARS compared to Prometheus and SMT. Clearly, larger randomized controlled trials are needed to resolve this issue.

Molecular Adsorbent Recirculation System and cerebral blood flow in acute on chronic liver failure

Raised intracranial pressure is a cause of death in patients with ALF. Although cerebral edema is rare in AoCLF, HE may result in significant morbidity with loss of airway protection, respiratory sepsis, and malnutrition. Accordingly, Schmidt and colleagues [13] assessed the effect of MARS on cerebral blood flow (CBF) in eight patients with AoCLF. Each patient received a single 10-hour MARS treatment. CBF was assessed by middle cerebral artery transcranial doppler imaging alone. This commonly used methodology has been previously validated using technecium-99 single-photon emission computed tomography in concert (not used in this study) [14]. In this cohort, CBF increased from baseline (mean velocity of 42 to $72 \mathrm{~cm} / \mathrm{s} ; p<0.05$ ). Clinically, the grade of clinical HE improved in three patients and remained unchanged in the other five. Significant decreases in bilirubin and arterial ammonia were also noted $(p<0.05)$. These results may reflect the ability of MARS to decrease intracerebral glutamine via reductions in ammonia [15]; however, studies to date have not assessed the effects of serial MARS treatments on CBF at more distant time endpoints.

MARS has demonstrated consistent benefit in $\mathrm{HE}$ in patients with AoCLF. Blei [16] randomly assigned 70 patients with grade 3 or $4 \mathrm{HE}$ to MARS $(n=39)$ and SMT $(n=31)$. The need for ventilation and the use of sedation were equal in both groups. Patients in the MARS group received therapy for 6 hours daily for 5 days or until a 2-grade improvement in HE was achieved. At day 5, 72\% of patients on MARS had achieved a 2 -grade improvement in $\mathrm{HE}$ versus $45 \%$ in the SMT group $(p=0.017)$.

Molecular Adsorbent Recirculation System and acute liver failure

There are limited controlled data examining the effect of MARS in ALF (Table 2). In 2003, Schmidt and colleagues [17] conducted a study to assess the effects of a single 6hour MARS treatment on hemodynamics, oxygen consumption, and biochemical profile in patients with ALF (HE grade $3 / 4$ developed within 7 days of symptoms). They randomly assigned 13 patients with ALF due to acetaminophen $(n=10)$, hepatitis $\mathrm{B}$ virus (HBV) $(n=2)$, and disulfram $(n=1)$. Eight received MARS therapy and five received SMT with cooling to match hypothermia induced by MARS. Oxygen consumption decreased by $22 \%(p<0.05)$ in the MARS group, whereas there was no significant change in the control group. SVRI increased by $46 \%$ in the MARS group during the 6 -hour run treatment versus a $6 \%$ increase in the controls $(p<0.0001)$. MAP also increased in the MARS 
Table 2

\begin{tabular}{|c|c|c|c|c|c|c|c|}
\hline \multirow[b]{2}{*}{ Study } & \multirow[b]{2}{*}{ Etiology } & \multirow[b]{2}{*}{ Number } & \multirow[b]{2}{*}{ Controlled } & \multicolumn{3}{|c|}{ Improvements } & \multirow[b]{2}{*}{ Survival (30 days) } \\
\hline & & & & Biochemical & CVS & CNS & \\
\hline Novelli et al. [39] & Fulminant & 9 & No & Yes & $\mathrm{N} / \mathrm{A}$ & $Y_{e s}^{a}$ & $66 \%$ \\
\hline Isoniemi et al. [40] & Toxic & 26 & No & Yes & N/A & N/A & $88 \%$ \\
\hline Tsai et al. [19] & Hepatitis $B$ virus & 10 & No & Yes & Yes & Yes & $30 \%$ \\
\hline Lee et al. [20] & Drug (herb) & 13 & No & Yes & N/A & No & $15 \%$ \\
\hline Lai et al. [21] & Drug/Non-A non-B hepatitis & 10 & No & No & $Y_{e s}^{b}$ & No & $30 \%$ \\
\hline Camus et al. [41] & Mixed & 23 & No & Yes $^{c}$ & N/A & Yes & N/A \\
\hline Schmidt et al. [17] & Hepatitis $B$ virus/Acetominophen & 13 & Yes & Yes $^{c}$ & Yes & $N / A$ & No $(62.5 \%$ versus $60 \%)$ \\
\hline El Banayosi et al. [18] & Ischemia & 27 & Yes & $\mathrm{No}^{d}$ & $\mathrm{~N} / \mathrm{A}$ & $N / A$ & Yes $(50 \%$ versus $32 \%)$ \\
\hline
\end{tabular}

Biochemical improvements: statistically significant reduction in bilirubin, bile acids, creatinine, and ammonia. aStatistics not provided; bstatistically significant after first but not second MARS treatment; ${ }^{\circ}$ only bilirubin and creatinine were statistically significant; ${ }^{d}$ non-significant improvement in bilirubin. CNS, improvement in hemodynamic parameters (mean arterial pressure, heart rate, vasopressor requirements); CNS, decrease in hepatic encephalopathy grade (neurological improvement); N/A, not assessed.

group $(p<0.001)$, whereas pressure was unchanged in controls. Compared to baseline, there were significant reductions in bilirubin, creatinine, and urea $(p<0.05)$ but not in ammonia in the MARS group. Survival was no different between the MARS and control patients.

Other studies have shown biochemical improvements with MARS in ALF without significant mortality benefit. In a controlled study of 27 patients treated for ALF due to cardiogenic shock, El Banayosy and colleagues [18] demonstrated non-significant reductions in conjugated and total bilirubin and mortality (Table 2). However, it is unclear whether this population truly met criteria for ALF, as there is no mention of grade of HE. In a case series of 10 patients with ALF due to HBV, Tsai and colleagues [19] demonstrated biochemical reductions (Table 2) at 3 months but mortality was $70 \%$ with one patient receiving a liver transplant. Lee and colleagues [20] have reported similar outcomes with MARS in a series of 13 patients with drug-induced ALF: a statistically significant reduction in total bilirubin after a single MARS treatment $(p<0.006)$ but an overall mortality of $85 \%$ at 20 days.

\section{Molecular Adsorbent Recirculation System and}

hemodynamics in acute liver failure

Lai and colleagues [21] reported 10 consecutive patients (paracetamol 8, non-A non-B hepatitis 1, and isoniazid 1) admitted with ALF and grade 3/4 HE who were treated with MARS for 8 hours on 2 consecutive days. During the first MARS treatment, there were significant increases in SVRI $(p=0.02)$ and cardiac index $(p=0.01)$. However, these changes were not maintained to the end of the second MARS session. No significant changes in MAP or intracranial pressure, as measured by reverse jugular bulb saturation, were noted. Bilirubin levels were not significantly decreased after MARS therapy. Three patients (30\%) survived; all had paracetamol hepatotoxicity and recovered without transplant.

\section{Molecular Adsorbent Recirculation System and inflammatory profile}

It has been suggested that MARS has a beneficial effect in patients with multi-organ dysfunction due to the inflammatory milieu present in liver failure. Guo and colleagues [22] reported an uncontrolled mixed cohort treated with MARS (not shown in Tables 1 and 2); 11 patients had ALF and 13 had AoCLF. Entry requirements included evidence of two or more of the following organ complications; HRS, HE (grade 2 or greater), disseminated intravascular coagulopathy, acute respiratory distress syndrome, variceal bleeding, sepsis, and cardiovascular failure. In total, the 24 patients received 66 treatments (6 to 24 hours per treatment) over the 7 days. Their results showed a statistically significant decrease in levels of nitric oxide, tumor necrosis factor-alpha (TNF- $\alpha$ ), interleukin (IL)-6, IL-8, and interferon-gamma $(p<0.01)$. Significant improvements were also noted in level of consciousness (Glasgow Coma Score of 7 to 13), renal function, respiratory function, as well as increase in MAP ( $p<0.01$ for all three comparisons). This study did not differentiate between ALF and AoCLF in subsequent analysis. The majority of patients had HBV (17/24).

Recently, Stadlbauer and colleagues [23] assessed cytokine levels in eight patients with AoCLF of diverse etiologies undergoing alternating treatments with MARS and Prometheus in a random crossover design. Thirty-four treatments (17 MARS and 17 Prometheus) were available for analysis. Although measurable plasma clearances were detected for IL-6, IL-8, IL-10, and TNF- $\alpha$, none was significant for MARS or Prometheus. Based on these studies, MARS does not 
Table 3

\begin{tabular}{|c|c|c|c|c|c|c|c|}
\hline \multirow[b]{2}{*}{ Study } & \multirow[b]{2}{*}{ Classification } & \multirow[b]{2}{*}{ Number } & \multirow[b]{2}{*}{ Controlled } & \multicolumn{3}{|c|}{ Improvements } & \multirow[b]{2}{*}{ Survival } \\
\hline & & & & Biochemical & CVS & CNS & \\
\hline Rifai et al. [28] & Acute on chronic liver failure & 11 & No & Yes & No & No & $28 \%$ at 30 days \\
\hline Skwarek et al. [31] & Acute liver failure & 13 & No & Yes & $\mathrm{N} / \mathrm{A}$ & N/A & $23 \%$ at 6 months \\
\hline Laleman et al. [12] & Acute on chronic liver failure (ethanol) & 18 & Yes & $Y_{e s}^{a}$ & No & N/A & $\mathrm{N} / \mathrm{A}$ \\
\hline
\end{tabular}

Biochemical improvements: statistically significant reduction in bilirubin, bile acids, creatinine, and ammonia. aBilirubin and bile acids only. CNS, improvement in hemodynamic parameters (mean arterial pressure, heart rate, vasopressor requirements); CNS, decrease in hepatic encephalopathy grade (neurological improvement); N/A, not assessed.

appear to have a significant impact on the inflammatory profile in AoCLF.

\section{Molecular Adsorbent Recirculation System and bleeding}

Blood coagulation is the result of a complex interaction between procoagulant, anticoagulant, and fibrinolytic proteins, many of which are synthesized by the liver. As previous liver support/perfusion systems have been associated with significant bleeding rates, two studies have studied coagulation and bleeding with MARS. Faybik and colleagues [24] described 33 patients undergoing 61 MARS treatments (15 with ALF, 8 with AoCLF, 3 with liver graft dysfunction, 5 with sepsis, and 2 with cholestasis). Although there was a statistically significant decrease in platelets and fibrinogen, platelet function as measured by thromboelastography was unaffected. Three moderate bleeding complications occurred requiring transfusions ( 3 to 4 units of packed cells), but none of the MARS runs had to be discontinued.

A second study by Doria and colleagues [25] examined 9 patients with cirrhosis who underwent MARS therapy: 6 with AoCLF and 3 with intractable pruritis. Four patients developed bleeding while on MARS: 3 from gastrointestinal sources and 1 from a genitourinary source. Four patients who bled during MARS treatments subsequently died. MARS resulted in a minor but statistically significant reduction in platelet count (37 to 32 per millilitre; $p<0.05$ ), worsened platelet function by thromboelastography $(p<0.05)$, and an increase in international normalised ratio for prothrombin time (1.9 to $3.5 ; p=$ not significant).

Clearly, patients with all forms of liver failure are at high risk for bleeding complications with or without MARS treatments. Controlled trials are necessary before the magnitude of these risks can be quantitated.

\section{Prometheus: Fractionated Plasma Separation and Adsorption}

Prometheus (Fresenius AG, Hamburg, Germany), or FPSA, was introduced in 1999. Its physiological basis is different than MARS; patient plasma is fractionated through an albumin-permeable filter with a cutoff of $250 \mathrm{kDa}$. Albumin and other plasma proteins cross the membrane and pass across two columns in series: one an anion-exchange column, another a neutral resin adsorber. The cleansed albumin/ plasma is returned to the standard blood pool circuit where it is then treated by conventional high-flux hemodialysis.

Early in vitro data showed that Prometheus effectively cleared bilirubin, bile acids, and aromatic amino acids [26]. Important compounds with a molecular weight of more than $250 \mathrm{kDa}$, such as fibrinogen, were unchanged. A recent study showed that in 18 patients with AoCLF (9 MARS and 9 Prometheus), greater reduction ratios in total bilirubin, conjugated bilirubin, and bile acids were obtained with Prometheus therapy than with MARS treatments with identical duration of therapy (5 hours), blood, and dialysate flow [27].

To date, no large controlled studies on Prometheus have been published but some small case series have been reported (Table 3). In 2003, Rifai and colleagues [28] reported 11 patients with AoCLF, HE, and HRS who were treated on 2 consecutive days (more than 4 hours) with Prometheus. Statistically significant reductions in conjugated and unconjugated bilirubin, bile acids, and ammonia levels were noted. Despite the drop in ammonia levels, no clinical change in HE was noted. Eight of 11 patients died, including one due to variceal bleeding. Rifai and colleagues [29] have also reported Prometheus use in 10 patients with HRS who underwent two consecutive Prometheus treatments. Statistically significant decreases in creatinine and urea levels and improvement in arterial blood $\mathrm{pH}$ were observed. This is not unexpected since conventional dialysis is incorporated into the circuit. Significant decreases in serum-conjugated bilirubin, bile acids, and ammonia levels were also noted. In the same patient population (AoCLF), Rifai and colleagues [30] have also shown that after two treatments, Prometheus did not significantly alter inflammatory cytokine levels (C reactive protein, IL-6, and TNF- $\alpha$ ).

Prometheus has also been used as a bridge to liver transplantation in patients with ALF. Skwarek and colleagues 
[31] reported 13 patients with ALF who received a total of 35 treatments lasting 6 to 10 hours. They noted statistically significant decreases in total bilirubin and ammonia. Improvements noted in acid-base balance and MAP did not reach statistical significance. Seven patients were bridged to liver transplantation, but only 3 were alive at 6 months. In summary, Prometheus does not appear to improve mortality in ALF unless transplant is performed, and even then, mortality is high.

The effect of Prometheus on systemic hemodynamics has not yet been established. Laleman and colleagues [12] compared the hemodynamic effects of Prometheus with MARS in 18 patients with AoCLF secondary to severe alcoholic hepatitis (Maddrey score above 60). Six patients received MARS, 6 received Prometheus, and 6 received SMT (including hemodialysis). After 3 consecutive days of therapy (mean approximately 6 hours), both MARS and Prometheus reduced serum bilirubin $(p<0.005)$, and MARS increased MAP $(\Delta+9 \mathrm{mmHg}$; $p<0.05)$ and SVRI $\left(\Delta+220\right.$ dyne-s $/ \mathrm{cm}^{5}$ per $\left.\mathrm{m}^{2} ; p<0.05\right)$ compared with Prometheus. No difference in hemodynamics was noted between Prometheus and SMT. Levels of endogenous norepinephrine, aldosterone, and vasopressin were reduced $(p<0.05)$ in the MARS group, whereas there was no statistically significant change in the Prometheus or SMT arms.

\section{Single-Pass Albumin Dialysis}

SPAD differs from MARS and Prometheus in that it uses a standard continuous venovenous hemodialysis (CVVHD) or venovenous hemodiafiltration system without any additional columns or circuits. Blood is dialysed against a standard dialysis solution with the addition of $4.4 \%$ albumin in the dialysate. The patient's blood is dialysed through a high-flux hollow-fibre hemodiafilter. In contrast to MARS, the albumin dialysate is not regenerated as no adsorbent columns are employed. A treatment is undertaken over 6 to 8 hours using 4.5 litres of dialysate and $700 \mathrm{ml}$ of albumin.

Sauer and colleagues [32] published in vitro work in which the efficiency of SPAD versus MARS versus standard CVVHD was determined from plasma samples obtained from 18 patients by plasmapheresis. Ammonia, bile acids, and conjugated bilirubin were added to the patient samples and then clearance was determined. This study showed that the removal of bile acids was similar between MARS and SPAD and that SPAD removed significantly more conjugated bilirubin than MARS (both significantly greater than CVVHD).

To date, little clinical data about SPAD have been published. Seige and colleagues [33] reported that HE grade improved in three patients with AoCLF and that one patient was successfully transplanted and survived. Kreymann and colleagues [34] reported that one patient with hyperacute liver failure secondary to Wilson disease was successfully bridged to transplantation and that SPAD efficiently cleared copper and bilirubin. Currently, there have been no rando- mized controlled studies showing its biochemical, hemodynamic, or survival benefits in vivo.

\section{Discussion}

There continues to be great interest in and potential for the various forms of extracorporeal albumin dialysis. However, at present, we cannot make an evidence-based recommendation supporting MARS, Prometheus, or SPAD. Of the three, MARS is the best-studied albumin dialysis technology in ALF and AoCLF; however, there is a paucity of robust randomized studies, and most of the data are largely observational. Although studies in both ALF and AoCLF have shown improvement (reduction) in biochemical markers (and HE grade in AoCLF) on MARS, no conclusive mortality benefit has been demonstrated to date, but studies are currently in progress to address this issue. While promising, hemodynamic improvement on MARS has not been demonstrated in all studies. Based on two studies [24,25], bleeding appears to be a significant potential issue with MARS therapy. Clearly, larger randomized studies are needed to definitively clarify issues of hemodynamics, mortality benefit, and side effects.

Prometheus has also demonstrated improvements in biochemical but not hemodynamic or inflammatory profiles. Prometheus does appear to be safe and has not been shown to increase bleeding complications in the limited uncontrolled studies. No mortality benefit to date has been demonstrated. A large, multi-centred randomized study in patients with AoCLF is currently under way to clarify its benefits and side effect profile. Given the paucity of data for SPAD, we cannot comment on its risks and benefits.

There are many methodological limitations in the published studies to date. First, many studies did not have homogeneous study groups (that is, ALF and AoCLF) or etiologies (each often has very different prognoses). Several studies did not stratify patients based on severity of illness (for example, Child Pugh score and APACHE [Acute Physiology and Chronic Health Evaluation] score) and hence it is difficult to assess patient matching or the impact of underlying disease on patient mortality with or without treatment. Duration and number of treatments varied between studies, which may explain inconsistent biochemical and hemodynamic findings. Some studies did not clearly define adjuvant therapies in either the albumin dialysis or SMT arms. Also, cointerventions such as transfusion targets, ventilatory strategies, hemodynamic targets, and algorithms for screening and treatment of infectious diseases were often not explicitly specified. Given this heterogeneity in previous studies, it is difficult to develop evidence-based clinical (for example, HE grade) and biochemical (for example, bilirubin) criteria to define the need to initiate therapy and furthermore define the optimal duration of therapy. These issues need to be addressed in larger controlled studies in order to truly assess the impact of albumin dialysis on mortality. 
One of the most important factors impacting the outcome of extracorporeal liver support is patient selection. At present, most patients being treated already have multi-organ failure and have been found to have a poor prognosis irrespective of MARS, Prometheus, or SPAD therapy (for example, many studies report a 30-day mortality of greater than 70\%). In previous epidemiological studies, Jalan and colleagues [35] have noted that patients with AoCLF with the presence of organ failure (that is, Sequential Organ Failure Assessment [SOFA] score above 8) have a distinctly worse prognosis than decompensated cirrhosis patients without organ failure. Furthermore, in these AoCLF patients with a SOFA score above 8 , those who previously decompensated in the previous 6 months have a much worse prognosis (mortality at 1 year of $78 \%$ versus 34\%) [35]. One could speculate that selecting decompensated cirrhosis patients and ALF patients prior to the development of multi-organ failure might prove to be more beneficial.

At present, bilirubin is the most studied biochemical endpoint of albumin dialysis therapy. However, since its decline does not necessarily correlate with survival or other important clinical endpoints such as bridging to liver transplant, other surrogate markers are sorely needed. Inderbitzin and colleagues [36] showed in post-surgical patients receiving MARS (either partial hepatectomy for hepatocellular carcinoma or cholangiocarcinoma or early graft dysfunction) that the most important prognostic markers for improvement on MARS were an indocyanine green plasma disappearance rate of above $5 \%$ and Factor $\mathrm{V}$ levels of above $30 \%$. This again suggests that earlier intervention will be most beneficial. This is an area that needs further exploration.

Regardless of the form of liver support, patient prognosis is universally poor if liver transplantation is not promptly available. Although a statistically significant 30-day survival benefit may be found, its clinical relevance, particularly in patients with AoCLF in the absence of transplant, is doubtful.

\section{Conclusion}

In summary, these new techniques using albumin dialysis represent potentially exciting advances in these desperately ill patients with ALF and AoCLF. The challenge upon us now is to learn how to best exploit these therapies to the patients' advantage. We need to determine which biochemical and clinical parameters correlate best with important patient outcomes such as overall survival, bridging to liver transplantation, and prevention of multi-organ failure. This information will help determine which technical refinements are most likely to be beneficial and can guide future clinical studies. Different patient subgroups are likely to have different benefits from albumin dialysis; trials should not combine ALF and AoCFL, and different etiologies will have to be evaluated separately. Adequately powered randomized controlled trials are now required to show the benefit of albumin dialysis. At this point, the appropriate comparator is still SMT rather than head-to-head comparisons of different forms of albumin dialysis in assessing short- and long-term survival, need for liver transplantation, and prevention of multiorgan failure.

\section{Competing interests}

CJK and JW are currently involved in a multi-centred study assessing Prometheus in patients with AoCLF but have not received any financial remuneration from Fresenius $A G$. NG, DK, and VGB declare that they have no competing interests.

\section{Authors' contributions}

CJK reviewed original articles, drafted the manuscript, and was involved in revising the manuscript. NG and JW reviewed original articles and were involved in revising the manuscript. DK outlined the format of the review and was involved in revising the manuscript. VGB initiated the review, reviewed original articles, and was involved in revising the manuscript. All authors read and approved the final manuscript.

\section{References}

1. Trey C, Davidson CS: The management of fulminant hepatic failure. Prog Liver Dis 1970, 3:282-298.

2. Sen S, Williams R, Jalan R: The pathophysiological basis of acute-on-chronic liver failure. Liver 2002, 22 Suppl 2:5-13.

3. Sen S, Williams R, Jalan R: Emerging indications for albumin dialysis. Am J Gastroentero/ 2005, 100:468-475

4. Evans TW: Review article: albumin as a drug-biological effects of albumin unrelated to oncotic pressure. Aliment Pharmacol Ther 2002, 16 Suppl 5:6-11.

5. O'Grady JG, Gimson AE, O'Brien CJ, Pucknell A, Hughes RD, Williams R: Controlled trials of charcoal hemoperfusion and prognostic factors in fulminant hepatic failure. Gastroentero/ogy 1988, 94(5 Pt 1):1186-1192.

6. Stange J, Ramlow W, Mitzner S, Schmidt R, Klinkmann H: Dialysis against a recycled albumin solution enables the removal of albumin-bound toxins. Artif Organs 1993, 17:809-813.

7. Stange J, Mitzner SR, Risler T, Erley CM, Lauchart W, Goehl H, Klammt S, Peszynski P, Freytag J, Hickstein H, et al.: Molecular adsorbent recycling system (MARS): clinical results of a new membrane-based blood purification system for bioartificial liver support. Artif Organs 1999, 23:319-330.

8. Mitzner SR, Stange J, Klammt S, Risler T, Erley CM, Bader BD, Berger ED, Lauchart W, Peszynski P, Freytag J, et al.: Improvement of hepatorenal syndrome with extracorporeal albumin dialysis MARS: results of a prospective, randomized, controlled clinical trial. Liver Transp/ 2000, 6:277-286.

9. Heemann U, Treichel U, Loock J, Philipp T, Gerken G, Malago M, Klammt S, Loehr M, Liebe S, Mitzner S, et al.: Albumin dialysis in cirrhosis with superimposed acute liver injury: a prospective, controlled study. Hepatology 2002, 36(4 Pt 1):949-958.

10. Schmidt LE, Sorensen VR, Svendsen LB, Hansen BA, Larsen FS: Hemodynamic changes during a single treatment with the molecular adsorbents recirculating system in patients with acute-on-chronic liver failure. Liver Transp/ 2001, 7:1034-1039.

11. Sen S, Davies NA, Mookerjee RP, Cheshire LM, Hodges SJ, Williams R, Jalan R: Pathophysiological effects of albumin dialysis in acute-on-chronic liver failure: a randomized controlled study. Liver Transp/ 2004, 10:1109-1119.

12. Laleman W, Wilmer A, Evenepoel P, Elst IV, Zeegers M, Zaman Z, Verslype C, Fevery J, Nevens F: Effect of the molecular adsorbent recirculating system and Prometheus devices on systemic haemodynamics and vasoactive agents in patients with acuteon-chronic alcoholic liver failure. Crit Care 2006, 10:R108.

13. Schmidt LE, Svendsen LB, Sorensen VR, Hansen BA, Larsen FS: Cerebral blood flow velocity increases during a single treatment with the molecular adsorbents recirculating system in patients with acute on chronic liver failure. Liver Transp/ 2001, 7:709-712. 
14. Uzuner N, Ak I, Gucuyener D, Asil T, Vardareli E, Ozdemir G: Cerebral hemodynamic patterns with technetium Tc $99 \mathrm{~m}$ exametazime single photon emission computed tomography and transcranial Doppler sonography: a validation study using visual stimulation. J Ultrasound Med 2002, 21:955-959.

15. Butterworth RF: Pathophysiology of hepatic encephalopathy: a new look at ammonia. Metab Brain Dis 2002, 17:221-227.

16. Blei A: Efficacy of Albumin Dialysis (MARS) in patients with cirrhosis and advanced grades of hepatic encephalopathy: a prospective controlled, randomized multicentred trial. Hepatology (AASLD Abstract) 2004, 40:726A.

17. Schmidt LE, Wang LP, Hansen BA, Larsen FS: Systemic hemodynamic effects of treatment with the molecular adsorbents recirculating system in patients with hyperacute liver failure: a prospective controlled trial. Liver Transp/ 2003, 9:290-297.

18. El Banayosy A, Kizner L, Schueler V, Bergmeier S, Cobaugh D, Koerfer R: First use of the Molecular Adsorbent Recirculating System technique on patients with hypoxic liver failure after cardiogenic shock. Asaio J 2004, 50:332-337.

19. Tsai MH, Chen YC, Wu CS, Ho YP, Fang JT, Lien JM, Yang C, Chu YY, Liu NJ, Lin CH, et al:: Extracorporal liver support with molecular adsorbents recirculating system in patients with hepatitis B-associated fulminant hepatic failure. Int $\mathrm{J}$ Clin Pract 2005, 59:1289-1294.

20. Lee KH, Lee MK, Sutedja DS, Lim SG: Outcome from molecular adsorbent recycling system (MARS) liver dialysis following drug-induced liver failure. Liver Int 2005, 25:973-977.

21. Lai WK, Haydon G, Mutimer D, Murphy N: The effect of molecular adsorbent recirculating system on pathophysiological parameters in patients with acute liver failure. Intensive Care Med 2005, 31:1544-1549.

22. Guo LM, Liu JY, Xu DZ, Li BS, Han H, Wang LH, Zhang WY, Lu LH, Guo X, Sun FX, et al.: Application of Molecular Adsorbents Recirculating System to remove NO and cytokines in severe liver failure patients with multiple organ dysfunction syndrome. Liver Int 2003, 23 Suppl 3:16-20.

23. Stadlbauer V, Krisper P, Aigner R, Haditsch B, Jung A, Lackner C, Stauber RE: Effect of extracorporeal liver support by MARS and Prometheus on serum cytokines in acute-on-chronic liver failure. Crit Care 2006, 10:R169.

24. Faybik $P$, Bacher A, Kozek-Langenecker SA, Steltzer H, Krenn $\mathrm{CG}$, Unger S, Hetz $\mathrm{H}$ : Molecular adsorbent recirculating system and hemostasis in patients at high risk of bleeding: an observational study. Crit Care 2006, 10:R24.

25. Doria C, Mandala L, Smith JD, Caruana G, Scott VL, Gruttadauria $\mathrm{S}$, Magnone M, Marino IR: Thromboelastography used to assess coagulation during treatment with molecular adsorbent recirculating system. Clin Transplant 2004, 18:365-371.

26. Falkenhagen D, Strobl W, Vogt G, Schrefl A, Linsberger I, Gerner FJ, Schoenhofen M: Fractionated plasma separation and adsorption system: a novel system for blood purification to remove albumin bound substances. Artif Organs 1999, 23:8186.

27. Evenepoel P, Laleman W, Wilmer A, Claes K, Kuypers D, Bammens B, Nevens F, Vanrenterghem Y: Prometheus versus molecular adsorbents recirculating system: comparison of efficiency in two different liver detoxification devices. Artif Organs 2006, 30:276-284.

28. Rifai K, Ernst T, Kretschmer U, Bahr MJ, Schneider A, Hafer C, Haller H, Manns MP, Fliser D: Prometheus-a new extracorporeal system for the treatment of liver failure. J Hepatol 2003, 39:984-990.

29. Rifai K, Ernst T, Kretschmer U, Hafer C, Haller H, Manns MP, Fliser D: The Prometheus device for extracorporeal support of combined liver and renal failure. Blood Purif 2005, 23:298302.

30. Rifai K, Ernst T, Kretschmer U, Haller H, Manns MP, Fliser D: Removal selectivity of Prometheus: a new extracorporeal liver support device. World J Gastroenterol 2006, 12:940-944.

31. Skwarek A, Grodzicki M, Nyckowski P, Kotulski M, Zieniewicz K, Michalowicz B, Patkowski W, Grzelak I, Paczkowska A, Giercuszkiewicz D, et al: : The use Prometheus FPSA system in the treatment of acute liver failure: preliminary results. Transplant Proc 2006, 38:209-211.

32. Sauer IM, Goetz M, Steffen I, Walter G, Kehr DC, Schwartlander R, Hwang YJ, Pascher A, Gerlach JC, Neuhaus P: In vitro comparison of the molecular adsorbent recirculation system
(MARS) and single-pass albumin dialysis (SPAD). Hepatology 2004, 39:1408-1414.

33. Seige M, Kreymann B, Jeschke B, Schweigart $U$, Kopp KF Classen M: Long-term treatment of patients with acute exacerbation of chronic liver failure by albumin dialysis. Transplant Proc 1999, 31:1371-1375.

34. Kreymann B, Seige M, Schweigart U, Kopp KF, Classen M: Albumin dialysis: effective removal of copper in a patient with fulminant Wilson disease and successful bridging to liver transplantation: a new possibility for the elimination of protein-bound toxins. J Hepatol 1999, 31:1080-1085.

35. Jalan R, Stadlbauer V, Sen S, Mookerjee RP, Davies NA, Hodges SJ, Zou Z, Cheshire LM, Williams R: Natural history of acute decompensation of cirrhosis: the basis of the definition, prognosis and pathophysiology of acute on chronic liver failure. Hepatology (AASLD Abstract) 2006, 44:371A.

36. Inderbitzin D, Muggli B, Ringger A, Beldi G, Gass M, Gloor B Uehlinger D, Regli B, Reichen J, Candinas D: Molecular absorbent recirculating system for the treatment of acute liver failure in surgical patients. J Gastrointest Surg 2005, 9:11551161; discussion 1161-1162.

37. Jalan R, Sen S, Steiner C, Kapoor D, Alisa A, Williams R: Extracorporeal liver support with molecular adsorbents recirculating system in patients with severe acute alcoholic hepatitis. $J$ Hepatol 2003, 38:24-31.

38. Di Campli C, Zocco MA, Gaspari R, Novi M, Candelli M, Santoliquido A, Flore R, Tondi P, Proietti R, Gasbarrini G, et al:: The decrease in cytokine concentration during albumin dialysis correlates with the prognosis of patients with acute on chronic liver failure. Transplant Proc 2005, 37:2551-2553.

39. Novelli G, Rossi M, Pretagostini R, Poli L, Novelli L, Berloco P, Ferretti G, lappelli M, Cortesini R: MARS (Molecular Adsorbent Recirculating System): experience in 34 cases of acute liver failure. Liver 2002, 22 Suppl 2:43-47.

40. Isoniemi H, Koivusalo AM, Repo H, llonen I, Hockerstedt K: The effect of albumin dialysis on cytokine levels in acute liver failure and need for liver transplantation. Transplant Proc 2005, 37:1088-1090.

41. Camus C, Lavoue S, Gacouin A, Le Tulzo Y, Lorho R, Boudjema $\mathrm{K}$, Jacquelinet $\mathrm{C}$, Thomas $\mathrm{R}$ : Molecular adsorbent recirculating system dialysis in patients with acute liver failure who are assessed for liver transplantation. Intensive Care Med 2006 , 32:1817-1825. 